\title{
Interacting Multiple Gaussian Particle Filter
}

\author{
Yanwen $\mathrm{Qu}$ \\ School of Computer Information and Engineer \\ Jiangxi Normal University \\ Nanchang, China
}

\begin{abstract}
Inspired by the framework of the Interacting Multiple Model (IMM), a method, called Interacting Multiple Gaussian Particle Filter (IMGPF), is proposed for solving the nonlinear Bayesian filtering problem with unknown continuous parameter. IMGPF regards the continuous parameter space as a union of disjoint subspaces, and each subspace is assigned to a model respectively. At each time step, for each model of IMGPF, under the assumption that the parameter belongs to the corresponding subspace, a Gaussian Particle Filter is applied to estimate the parameter and the state together. The parameter of each model of IMM is a fixed value, while the parameter of each model of IMGPF is a random variable need to be estimated. Thus IMGPF can achieve better estimation performance than IMM when the true parameter does not close to any element of the IMM model set. A simulation example of bearings only tracking problem is presented to demonstrate the effectiveness of IMGPF.
\end{abstract}

Keywords;Interacting multiple model; Particle Fiter; Gaussian Particle Filter

\section{INTRODUCTION}

In the past years, there has been much interest in the nonlinear Bayesian filtering problem with unknown parameter. As far as we known, the following three kinds of Bayesian filtering methods are widely used to deal with such a problem:

1) Extended Kalman Filter(EKF) or Unscented Kalman Filter $(U K F)$ based methods ${ }^{[1,2]}$. In this kind of method, the unknown parameter time series is assumed to be a random walk affected by the artificial process noise. The variance of the process noise is assumed to be large if the true parameter may jump greatly at each time step, while it is assumed to be small if the true parameter may jump slightly or remain static at each time step. This kind of method is characterized by its fast calculation and easy implementation; however, its estimation performance may get worse when the real parameter process is complex.

2) Particle Filter ${ }^{\mathrm{s}[3]}(\mathrm{PF})$ based methods: In this kind of method, various sophisticated stochastic process can be used to model the parameter process, such as random walk ${ }^{[4]}$, kernel smoothing ${ }^{[5,6]}$, time homogeneous Markov Chain ${ }^{[7-9]}$, etc. The PF based method has been widely applied in many cases in the last decade, however, a small number of particles may degrade the estimation performance of the PFs based methods. For example, suppose that the true parameter jumps into a region, which is a small probability event, a small particle number will led to a case that the parameter component of each particle will far from the true parameter.

3) Interacting Multiple Mode ${ }^{1[10]}$ (IMM) based methods $s^{[7,9 \text {, }}$ ${ }^{11]}$ : IMM has shown its great success in handling problems with both structural and parameter uncertainties in recent years. IMM adopts a model set $\Omega_{\mathrm{IMM}}$, which is a collection of discrete parameter points, to approximate the parameter space, and each elements of $\Omega_{\mathrm{IMM}}$ is assigned to a model. The parameter process is assumed to be a first order Markov process on the $\Omega_{\text {IMM. }}$. At each time step, IMM runs a filter for each model. Many kinds of filters can be used for the model of IMM, such as $\operatorname{EKF}^{[7,9]}, \mathrm{UKF}^{[7,9]}$ and Raoblackwellised Unscented Kalman Filter $^{[11]}$. The estimation performance of the IMM based methods is influenced by the $\Omega_{\mathrm{IMM}}$. As soon as the parameter jumps, no matter how large the variation is, this kind of method are adapted to the change rapidly as long as the new parameter is close to any element of $\Omega_{\mathrm{IMM}}$. However, such condition might appear hard to satisfy provided the parameter space is continuous and the cardinality of the $\Omega_{\mathrm{IMM}}$ is small.

In this work we attempt to develop an improved Interacting Multiple Model for the nonlinear Bayesian filtering problem with unknown continuous parameter, named Interacting Multiple Gaussian Particle Filter (IMGPF). It achieves better estimation performance than IMM when the true parameter is not close to any element of the $\Omega_{\mathrm{IMM}}$.

\section{State Space Model}

Consider the following state space model affected by the system parameter.

$$
\begin{aligned}
& x_{t}=f\left(x_{t-1}, v_{t}, \theta_{t}\right) \\
& y_{t}=h\left(x_{t}, w_{t}, \theta_{t}\right)
\end{aligned}
$$

where, the subscript $t$ is the time index. $f()$ and $h()$ are two known nonlinear functions of the state $x_{t} \in \mathrm{R}^{n x} . y_{t}$ is the measurement, and $y_{1: t}=\left\{y_{1}, \ldots, y_{t}\right\}$ denotes the measurement sequence from time 1 to time $t . \theta_{t} \in \Omega_{\theta} \subseteq \mathrm{R}^{\mathrm{n} \theta}$ is the parameter, and $\Omega_{\theta}$ denotes the continuous parameter space. The parameter process is assumed unknown. The process noise $v_{t}, t=0,1,2, \ldots$ and the measurement noise $w_{t}, t=1,2, \ldots$ are independent random variables.

The state transition probability distribution function (pdf) $p\left(\mathrm{~d} x_{t} \mid x_{t-1}, \theta_{t}\right)$ and the measurement likelihood $p\left(y_{t} \mid x_{t}, \theta_{t}\right)$ can be derived from (1) and (2).

We will discuss below how to estimate the combination state $\eta_{\mathrm{t}}=\left[x_{\mathrm{t}}, \theta_{\mathrm{t}}\right]$ which includes the state and the parameter. 


\section{IMGPF INTRODUCTION}

\section{A. Parameter Process Modeling}

The IMGPF model set $\Omega_{\mathrm{IMGPF}}=\left\{\mathrm{s}_{(i)}, i=1, \ldots, \mathrm{N}_{\mathrm{IMGPF}}\right\}$ is a partition of the parameter space, where $\mathrm{s}_{(i)}$ is a subspace of the $\Omega_{\theta}, i=1, \ldots, \mathrm{N}_{\text {IMGPF. }}$. At each time step, $\theta_{t}$ belongs to one and only one element of the $\Omega_{\mathrm{IMGPF}}$, denoted as $s_{t}$. Let $s_{t}^{(i)}$ represent the event: $s_{t}=\mathrm{s}_{(i)}, i=1, \ldots, \mathrm{N}_{\mathrm{IMGPF}}$.

For the purpose of modeling different kinds of parameter behaviors, such as jumping and being static, effectively, the parameter process is assumed to obey the following hypotheses

- $\left\{s_{t}, t \in \mathrm{N}^{+}\right\}$is a first order Markov process with transition probability matrix (TPM) $\pi_{\mathrm{IMGPF}}$. The element in the $i$ th row and $j$ th column of $\pi_{\mathrm{IMGPF}}$ is denoted as $\pi\left(s_{(i)} \mid s_{(j)}\right)$.

- If $s_{t}=s_{t-1}$, the parameter remains static with probability $\mathrm{c}\left(s_{t}\right)$. The probability $\mathrm{c}\left(s_{t}\right)$ is called inner subspace conditional static probability.

- If $s_{t}=s_{t-1}$, the parameter jumps from a point in $s_{t}$ to another one in $s_{t}$ (include the event that $\theta_{t}=\theta_{t-1}$ ) with probability $\left(1-\mathrm{c}\left(s_{t}\right)\right)$, and the transition probability distribution function (PDF) is $\mathrm{q}\left(\mathrm{d} \theta_{t} \mid s_{t}, s_{t-1}\right)$.

- If $s_{t} \neq s_{t-1}$, the parameter jumps from a point in $s_{t-1}$ to a point in $s_{t}$ with the transition PDF $\mathrm{q}\left(\mathrm{d} \theta_{t} \mid s_{t}, s_{t-1}\right)$.

- $\mathrm{q}\left(\mathrm{d} \theta_{t} \mid s_{t}, s_{t-1}\right)$ is the uniform distribution over $s_{t}$.

\section{B. Model Set Design}

In theory, any partition of the $\Omega_{\theta}$ can be used as the IMGPF model set. Inspired by the fifth hypothesis mentioned above, one optional design criterion of the model set is to minimize the following function,

$$
\sum_{i=1}^{\mathrm{N}_{\mathrm{IMGF}}}\left(P_{s}^{(i)} \int_{\theta \in s_{(i)}} \mathrm{d} \theta\right) /\left(\int_{\theta \in \Omega_{\theta}} \mathrm{d} \theta\right)
$$

where

$$
\begin{aligned}
& P_{s}^{(i)}=\int_{\theta \in s_{(i)}}\left(\left(\theta-m_{s}^{(i)}\right)\left(\theta-m_{s}^{(i)}\right)^{T}\right) /\left(\int_{\phi \in s_{(i)}} \mathrm{d} \phi\right) \mathrm{d} \theta \\
& m_{s}^{(i)}=\int_{\theta \in s_{(i)}} \theta /\left(\int_{\phi \in s_{(i)}} \mathrm{d} \phi\right) \mathrm{d} \theta
\end{aligned}
$$

$m_{s}{ }^{(i)}$ and $P_{s}^{(i)}$ are the mean and covariance of the uniform distribution over the $s_{(i)}$, respectively.

Equation (3) implies that the k-means clustering algorithm $^{[12]}$ can be used to design the IMGPF model set. The main steps of a numerical approach to minimize the (3) are: (a) drawing a set of samples from the $\Omega_{\theta}$ equally by using Monte Carlo method; (b) using k-means clustering method to decompose the samples into $\mathrm{N}_{\mathrm{IMGPF}}$ clusters, which are used to approximate the elements of the IMGPF model set, respectively; (c) computing the sample mean and covariance of the $i$ th cluster to approximate the $m_{s}{ }^{(i)}$ and $P_{s}^{(i)}$, respectively.

\section{Basic idea of IMGPF}

For the purpose of responding to the parameter jump as soon as possible, IMGPF inherits the idea of parallel computation from IMM.

IMGPF assigns each subspace to a model respectively. For each model, under the assumption that the parameter belongs to the corresponding sub-space, a Gaussian particle filter ${ }^{[13]}$ (GPF) is employed to estimate the state and the parameter together. The estimations of all the GPFs will be combined to generate the estimation of the IMGPF.

The main difference between IMM and IMGPF is that the parameter of each model of IMM is a constant point, however, that of IMGPF is an unknown parameter needs to be estimated. Thus, the IMGPF may perform better than IMM when the true parameter is not close to any elements of the IMM model set.

\section{Main steps of IMGPF}

Some notations are defined below to facilitate the readability of the paper:

- $p\left(s_{t}^{(i)} \mid y_{1: t-1}\right)$ : conditional probability of the true parameter belongs to the $i$ th model at time $t$ given the data $y_{1: t-1}$, named the prior probability of the $i$ th model.

- $p\left(s_{t}^{(i)} \mid y_{1: t}\right)$ : conditional probability of the true parameter belongs to the $i$ th model at time $t$ given the data $y_{1: t}$, named the posterior probability of the $i$ th model.

- $p\left(y_{t} \mid s_{t}^{(i)}, y_{1: t-1}\right)$ : conditional probability of the measurement $y_{t}$ given the event $s_{t}^{(i)}$ and the date $y_{1: t}$, named the likelihood of the $i$ th model.

- $\quad p\left(\mathrm{~d} \eta_{\mathrm{t}} \mid s_{t}^{(i)}, y_{1: t-1}\right)$ : conditional pdf of the combination state $\eta_{\mathrm{t}}$ given the event $s_{t}^{(i)}$ and the data $y_{1: t-1}$ :

- $\quad N\left(\mathrm{~d} \eta_{\mathrm{t}} ; m_{\eta, t}^{(i)} ; P_{\eta \eta, t}^{(i)}\right)$ : a Gaussian distribution which is used to approximate the $p\left(\mathrm{~d} \eta_{\mathrm{t}} \mid s_{t}^{(i)}, y_{1: t-1}\right)$, named the output pdf of the $i$ th model.

- $\psi_{t}=\left[x_{t-1}, \theta_{t}\right]:$ a vector which includes the $x_{t-1}$ and $\theta_{t}$, named the mixed state.

- $\quad p\left(\mathrm{~d} \psi_{\mathrm{t}} \mid s_{t}^{(i)}, y_{1: t-1}\right)$ : conditional pdf of the $\psi_{\mathrm{t}}$ given the event $s_{t}^{(i)}$ and data $y_{1: t-1}$.

- $\quad N\left(\mathrm{~d} \psi_{\mathrm{t}} ; m_{\psi, t}^{(i)} ; P_{\psi \psi, t}^{(i)}\right):$ a Gaussian distribution which is used to approximate the $p\left(\mathrm{~d} \psi_{\mathrm{t}} \mid s_{t}^{(i)}, y_{1: t-1}\right)$, named the input pdf of the $i$ th model

Assuming that $p\left(s_{t-1}^{(i)} \mid y_{1: t-1}\right)$ and $N\left(\mathrm{~d} \eta_{t-1} ; m_{\eta, t-1}^{(i)} ; P_{\eta \eta, t-1}^{(i)}\right)$ of the $i$ th model, $i=1, \ldots, \mathrm{N}_{\mathrm{IMGPF}}$, have been calculated before the time $t$, where

$$
\begin{aligned}
& m_{\eta, t-1}^{(i)}=\left[m_{x, t-1}^{(i)}, m_{\theta, t-1}^{(i)}\right] \\
& P_{\eta \eta, t-1}^{(i)}=\left[\begin{array}{ll}
P_{x x, t-1}^{(i)} & P_{x \theta, t-1}^{(i)} \\
P_{\theta x, t-1}^{(i)} & P_{\theta \theta, t-1}^{(i)}
\end{array}\right]
\end{aligned}
$$


The $m_{x, t-1}^{(i)}$ is a component of $m_{\eta, t-1}^{(i)}$, which is about the mean of the state. The $P_{x x, t-1}^{(i)}$ is a component of $P_{\eta \eta, t-1}^{(i)}$, which is about the covariance of the state.

The IMGPF has four major steps associated with it.

First, for $i=1, \ldots, \mathrm{N}_{\mathrm{IMGPF}}$, computer the prior probability of the $i$ th model by

$$
p\left(s_{t}^{(i)} \mid y_{1: t-1}\right)=\sum_{j=1}^{\mathrm{N}_{\mathrm{IMGPF}}} \pi\left(s_{(i)} \mid s_{(j)}\right) p\left(s_{t-1}^{(j)} \mid y_{1: t-1}\right)
$$

Second, for $i=1, \ldots, \mathrm{N}_{\text {IMGPF, a GPF is used as the model }}$ filter to compute the $p\left(y_{t} \mid s_{t}^{(i)}, y_{1: t-1}\right)$ and $N\left(\mathrm{~d} \eta_{\mathrm{t}} ; m_{\eta, t}^{(i)} ; P_{\eta \eta, t}^{(i)}\right)$. The $m_{\eta, t}^{(i)}$ is regarded as the estimation of the $i$ th model. More details about this step will be discussed in the followed section.

Third, for $i=1, \ldots, \mathrm{N}_{\mathrm{IMGPF}}$, update the posterior probability of the $i$ th model by

$$
p\left(s_{t}^{(i)} \mid y_{1: t}\right)=\frac{p\left(y_{t} \mid s_{t}^{(i)}, y_{1: t-1}\right) p\left(s_{t}^{(i)} \mid y_{1: t-1}\right)}{\sum_{j=1}^{N_{\text {IMGPF }}} p\left(y_{t} \mid s_{t}^{(j)}, y_{1: t-1}\right) p\left(s_{t}^{(j)} \mid y_{1: t-1}\right)}
$$

Fourth, obtain the IMGPF estimation by

$$
e_{\mathrm{IMGPF}}\left[\eta_{t}\right]=\sum_{i=1}^{\mathrm{N}_{\mathrm{IMGPF}}} \mathrm{m}_{\eta, t}^{(i)} p\left(s_{t}^{(i)} \mid y_{1: t}\right)
$$

\section{E. Model filtering}

The $i$ th model is taken as an example to show how the model filter works. There are four steps in the process of model filtering, which are given as follows:

Step1) Input pdf calculation: Compute the mean and covariance of $N\left(\mathrm{~d} \psi_{\mathrm{t}} ; m_{\psi, t}^{(i)} ; P_{\psi \psi, t}^{(i)}\right)$ by

$$
\begin{aligned}
m_{\psi, t}^{(i)}= & m_{\eta, t-1}^{(i)} c\left(s_{(i)}\right) q\left(s_{t-1}^{(i)} \mid s_{t}^{(i)}\right)+\sum_{j \neq i} m_{\psi, t-1}^{*(i, j)} q\left(s_{t-1}^{(j)} \mid s_{t}^{(i)}\right) \\
& +m_{\psi, t-1}^{*(i, i)}\left(1-c\left(s_{(i)}\right)\right) q\left(s_{t-1}^{(i)} \mid s_{t}^{(i)}\right)
\end{aligned}
$$

$$
\begin{aligned}
P_{\psi \psi, t}^{(i)}= & \left(m_{\eta, t-1}^{(i)}-m_{\psi, t}^{(i)}\right)\left(m_{\eta, t-1}^{(i)}-m_{\psi, t}^{(i)}\right)^{T} c\left(s_{(i)}\right) q\left(s_{t-1}^{(i)} \mid s_{t}^{(i)}\right) \\
& +\left(m_{\psi, t-1}^{*(i, i)}-m_{\psi, t}^{(i)}\right)\left(m_{\psi, t-1}^{*(i, i)}-m_{\psi, t}^{(i)}\right)^{T}\left(1-c\left(s_{(i)}\right)\right) q\left(s_{t-1}^{(i)} \mid s_{t}^{(i)}\right) \\
& +\sum_{j \neq i}\left(m_{\psi, t-1}^{*(i, j)}-m_{\psi, t}^{(i)}\right)\left(m_{\psi, t-1}^{*(i, j)}-m_{\psi, t}^{(i)}\right)^{T} q\left(s_{t-1}^{(j)} \mid s_{t}^{(i)}\right) \\
& +P_{\eta \eta, t-1}^{(i)} c\left(s_{(i)}\right) q\left(s_{t-1}^{(i)} \mid s_{t}^{(i)}\right)+P_{\psi \psi, t-1}^{*(i, i)}\left(1-c\left(s_{(i)}\right)\right) q\left(s_{t-1}^{(i)} \mid s_{t}^{(i)}\right) \\
& +\sum_{j \neq i} P_{\psi \psi, t-1}^{*(i, j)} q\left(s_{t-1}^{(j)} \mid s_{t}^{(i)}\right)
\end{aligned}
$$

where

$$
q\left(s_{t-1}^{(j)} \mid s_{t}^{(i)}\right)=\frac{\pi\left(s_{(i)} \mid s_{(j)}\right) p\left(s_{t-1}^{(j)} \mid y_{1: t-1}\right)}{\sum_{k=1}^{\mathrm{N}_{\mathrm{IMGF}}} \pi\left(s_{(i)} \mid s_{(k)}\right) p\left(s_{t-1}^{(k)} \mid y_{1: t-1}\right)}
$$

$$
\begin{gathered}
m_{\psi, t-1}^{*(i, j)}=\left[m_{x, t-1}^{(j)}, m_{s}^{(i)}\right] \\
P_{\psi \psi, t-1}^{*(i, j)}=\left[\begin{array}{cc}
P_{x x, t-1}^{(j)} & 0 \\
0 & P_{s}^{(i)}
\end{array}\right]
\end{gathered}
$$

Step2) Filtering: A GPF is used to estimate the likelihood and the output pdf the ith submodel.

Step2.1) Draw particles from $N\left(\mathrm{~d} \psi_{\mathrm{t}} ; m_{\psi, t}^{(i)} ; P_{\psi \psi, t}^{(i)}\right)$, and denote them as $\left\{\psi_{t}^{(i, j)}=\left[x_{t-1}^{(i, j)}, \theta_{t}^{(i, j)}\right], j=1, \ldots, \mathrm{N}_{\mathrm{s}}\right\}$.

Step2.2) For $j=1, \ldots, \mathrm{N}_{\mathrm{s}}$, sample from $p\left(\mathrm{~d} x_{t} \mid x_{t-1}^{(i, j)}, \theta_{t}^{(i, j)}\right)$ to obtain the $x_{t}^{(i, j)}$, and denote $\eta_{t}^{(i, j)}=\left[x_{t}^{(i, j)}, \theta_{t}^{(i, j)}\right]$,

Step2.3) Approximate the likelihood of the $i$ th model by

$$
p\left(y_{t} \mid s_{t}^{(i)}, y_{1: t-1}\right)=\sum_{j=1}^{\mathrm{N}_{\mathrm{s}}} p\left(y_{t} \mid x_{t}^{(i, j)}, \theta_{t}^{(i, j)}\right) / \mathrm{N}_{\mathrm{s}}
$$

Step2.4) Estimate the mean and covariance of $N\left(\mathrm{~d} \eta_{\mathrm{t}} ; m_{\eta, t}^{(i)} ; P_{\eta \eta, t}^{(i)}\right)$ by

$$
\begin{gathered}
m_{\eta, t}^{(i)}=\sum_{j=1}^{\mathrm{Ns}} \eta_{t}^{(i, j)} p\left(y_{t} \mid x_{t}^{(i, j)}, \theta_{t}^{(i, j)}\right) /\left(\mathrm{N}_{\mathrm{s}} p\left(y_{t} \mid s_{t}^{(i)}, y_{1: t-1}\right)\right) \\
P_{\eta \eta, t}^{(i)}=\frac{\sum_{j=1}^{\mathrm{Ns}}\left(\eta_{t}^{(i, j)}-m_{\eta, t}^{(i)}\right)\left(\eta_{t}^{(i, j)}-m_{\eta, t}^{(i)}\right)^{T} p\left(y_{t} \mid x_{t}^{(i, j)}, \theta_{t}^{(i, j)}\right)}{\mathrm{N}_{\mathrm{s}} p\left(y_{t} \mid s_{t}^{(i)}, y_{1: t-1}\right)}
\end{gathered}
$$

Step3) Reinitialize output pdf: If the following inequality is not satisfied,

$$
\left(m_{\eta, t}^{(i)}-m_{s}^{(i)}\right)^{T} P_{s}^{(i)}\left(m_{\eta, t}^{(i)}-m_{s}^{(i)}\right) \leq \mathrm{Th}
$$

the mean and covariance of the output pdf should be reinitialized as:

$$
\begin{gathered}
\tilde{m}_{\eta, t}^{(i)}=\left[m_{x, t}^{(i)}, m_{s}^{(i)}\right] \\
\tilde{P}_{\eta \eta, t}^{(i)}=\left[\begin{array}{ll}
P_{x x, t}^{(i)} & \\
& P_{s}^{(i)}
\end{array}\right]
\end{gathered}
$$

where Th is a threshold.

The reason to carry out the Step3 is that the mean of the output pdf may away from $s_{(i)}$ if the true parameter is not belongs to the $s_{(i)}$ for a long time. However, if that happens, it may disobey the basic idea of the IMGPF, because the $i$ th model concerns about the distribution over the $s_{(i)}$. One simple approach to solve such an issue is to test the (19) to determine whether the output pdf should be reinitialized according to (20) and (21) or not. More sophisticated methods will be taken into account in our future work.

\section{SimUlation RESUlts}

The proposed IMGPF is compared with IMM, SIR and UKF under a simulation of bearings only tracking problem. 
Suppose the object moves in the X-Y plane according the constant turn ${ }^{[14]}$ (CT) model, and the measurements of the object taken by the sensors at fixed intervals are the bearings (subject to Gaussian white noise) with respect to three sensors. The sate space form of the dynamic system is as follows:

$$
\begin{aligned}
& x_{t}=\left[\begin{array}{cccc}
1 & \frac{\sin \theta_{t}}{\theta_{t}} & 0 & \frac{\cos \theta_{t}-1}{\theta_{t}} \\
0 & \cos \theta_{t} & 0 & -\sin \theta_{t} \\
0 & \frac{1-\cos \theta_{t}}{\theta_{t}} & 1 & \frac{\sin \theta_{t}}{\theta_{t}} \\
0 & \sin \theta_{t} & 0 & \cos \theta_{t}
\end{array}\right]+\left[\begin{array}{cc}
0.5 & 0 \\
1 & 0 \\
0 & 0.5 \\
0 & 1
\end{array}\right] v_{t} \\
& y_{t}=\left[\begin{array}{l}
\tan ^{-1}\left[\left(y_{t}^{c}-0\right) /\left(x_{t}^{c}-0\right)\right] \\
\tan ^{-1}\left[\left(y_{t}^{c}-0.2\right) /\left(x_{t}^{c}-0.2\right)\right] \\
\tan ^{-1}\left[\left(y_{t}^{c}+0.2\right) /\left(x_{t}^{c}-0.2\right)\right]
\end{array}\right]+w_{t}
\end{aligned}
$$

where, $x_{t}=\left[x_{t}^{c}, x_{t}^{v}, y_{t}^{c}, y_{t}^{v}\right] . x_{t}^{c}$ and $y_{t}^{c}$ denote the Cartesian coordinates of the object, and $x_{t}^{v}$ and $y_{t}^{v}$ denotes the velocities in the $\mathrm{X}$ and $\mathrm{Y}$ directions, respectively. $v_{t}$ and $w_{t}$ are zero-mean white noise with covariance $P_{v v}=\left(0.001\left(\mathrm{~m} / \mathrm{s}^{2}\right)\right)^{2} \times I_{2}$ and $P_{w w}=(0.005(\mathrm{rad}))^{2} \times I_{3}$, respectively, where $I_{\mathrm{n}}$ is the $\mathrm{n} \times \mathrm{n}$ identity matrix. The initial pdf of $x_{0}$ is a Gaussian distribution with mean $[0(\mathrm{~m}), 0(\mathrm{~m} / \mathrm{s}), 0.4(\mathrm{~m}),-0.005(\mathrm{~m} / \mathrm{s})]$ and covariance $\operatorname{diag}\left[(0.005(\mathrm{~m}))^{2} ;(0.001(\mathrm{~m} / \mathrm{s}))^{2} ;(0.005(\mathrm{~m}))^{2} ;(0.001(\mathrm{~m} / \mathrm{s}))^{2}\right]$.

The turn rate (parameter) time series which is unknown to the filters is illustrated in Table.1

TABLE I. TURN RATE AT EACH TIME STEP

\begin{tabular}{|l|c|}
\hline \multicolumn{1}{|c|}{ Time(s) } & Turn rate( $\left.{ }^{\circ} / \mathbf{s}\right)$ \\
\hline $1-10$ & 0 \\
\hline $11-15$ & 18 \\
\hline $16-25$ & 33 \\
\hline $26-30$ & -33 \\
\hline
\end{tabular}

IMGPF and IMM both include two models, and the initial prior probability of each model is 0.5 . The IMM model set is $\Omega_{\mathrm{IMM}}=\{-22.5 \times \pi / 180,22.5 \times \pi / 180\}$, and the IMGPF model set is $\Omega_{\mathrm{IMGPF}}=\left\{s_{(1)}=\{\theta \mid-45 \times \pi / 180 \leq \theta<0\}, s_{(2)}=\{\theta \mid 0 \leq \theta \leq 45 \times \pi / 180\}\right\}$. IMM and JSIMM have the same model TPM:

$$
\Pi=\left[\begin{array}{ll}
0.9 & 0.1 \\
0.1 & 0.9
\end{array}\right]
$$

Let $\mathrm{c}\left(s_{(1)}\right)=\mathrm{c}\left(s_{(2)}\right)=0.9$. The SIR uses 400 particles to estimate the state and parameter together.

A simulation with 100 Monte Carlo runs is conducted to test the performance. The root mean square error (RMSE) of position and parameter estimation for the four methods are show in Fig.1 and Fig.2, respectively.

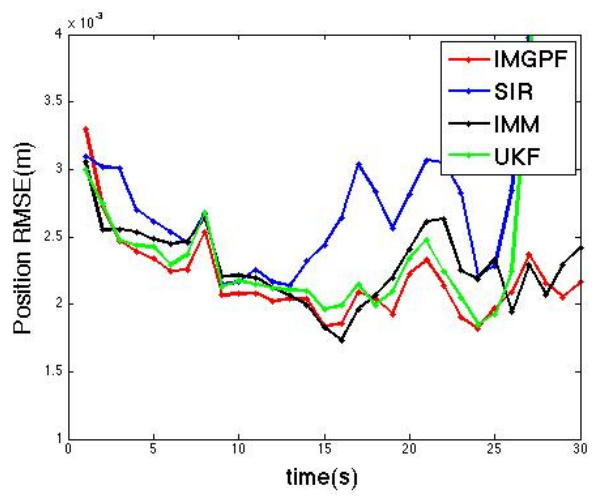

Figure 1. Root mean square error of position estimation

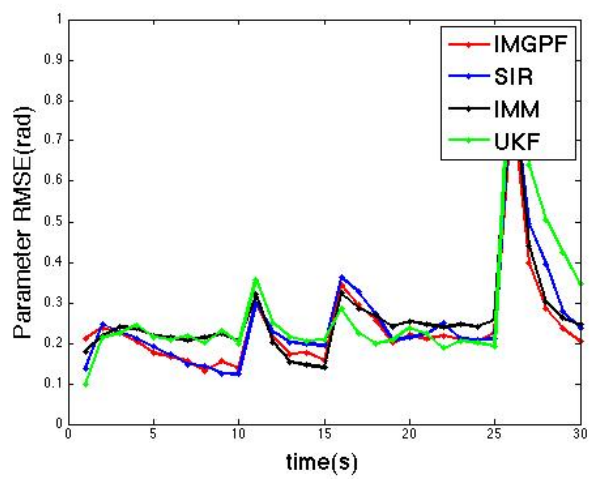

Figure 2. Root mean square error of parameter estimation

As is shown in the Fig.1 and Fig.2 that when the true parameter is close to any one of the elements in the IMM model set, the IMM performs best and IMGPF is the next. However, the estimation performance of IMM gets worse when none of the elements in the IMM model set is close to the true parameter, such as the period between the $1(\mathrm{~s})$ to $10(\mathrm{~s})$. Meanwhile, the IMM performs better than other methods.

Parameter change occurs three times in the simulation. When the parameter jumps greatly, such as the change happening between 25(s) to 26(s), the SIR and UKF fail to response to the large change as the particle number of SIR is insufficient and the variance of the process noise of the UKF is not large.

\section{CONCLUSION}

The estimation performance of IMM may degrade when the true parameter is not close to any element of the IMM model set. The main purpose of this study is to develop an improved method to solve such a problem.

The IMGPF proposed in this paper inherits the idea of parallel computation from IMM. The main difference between IMM and IMGPF is that the parameter of each model of IMM is a constant point, however, that of IMGPF is an unknown parameter needs to be estimated

The simulation results show that when compared with IMM, SIR and UKF, our new approach performs better in many situations 


\section{REFERENCES}

[1] van der Mermer R, Wan E A. The square-root unscented kalman filter for state and parameter estimation. Proc. of the 2001 IEEE International Conf. on Acoustics, Speech and Signal Processing-Proceedings. Salt Lake City: IEEE Press, 2001.3461-3464.

[2] Ljung L. Asymptotic behavior of the extended kalman filter as a parameter estimator for linear systems IEEE Transactions on Automatic Control, 1979, AC-24(1):36-50.

[3] Gordon N, Salmond D, Smith A F. Novel approach to nonlinear/nongaussian bayesian state estimation. IEE Proceedings Part F Radar and Signal Processing, 1993, 140 2):107-113.

[4] Kitagawa G. A self-organizing state-space model. Journal of American Statistical Association, 1998, 93: p 1203-1215.

[5] Liu J, West M. Combined parameter and state estimation in simulationbased filtering. In: Doucet, de Freitas N, and Gordon N, Eds. Sequential Monte Carlo in Practice. New York: Springer-Verlag, 2001:197-223.

[6] Chen T, Morris J, Martin E. Particle filters for state and parameter estimation in batch processes. Journal of Process Control, 2005, 15(6): 665-673.
[7] Ristic B, Arulampalam M S. Tracking a manoeuvring target using angleonly measurements: algorithms and performance. Signal Processing, 2003, 83(6): 1223-1238.

[8] McGinnity S, Irwin G W. Multiple model bootstrap filter for maneuvering target tracking. IEEE Transactions on Aerospace and Electronic Systems, 2000, 36(3): 1006-1012.

[9] Cui N Z, Hong L, Layne J R. A comparison of nonlinear filtering approaches with an application to ground target tracking. Signal Processing, 2005, 85(8): 1469-1492.

[10] Blom H A P, Bar-Shalom Y. The interacting multiple model algorithm for systems with Markovian switching coefficients. IEEE Transcations on Automatic Control, 1988, 33(8): 780-783.

[11] Zhong Z W, Meng H D, Wang X Q. Extended target tracking using an IMM based rao-blackwellised unscented kalman Filter. Proc. of the 2008 9th International Conf. on Signal Processing. Beijing: IEEE Press, 2008. 2409-2412

[12] Duda R O, Hart P E, Stork D G. Pattern Classification, Second Edition. Beijing: Machine Press, 2004.

[13] Kotecha J H, Djuric P M, Gaussian Particle Filtering, IEEE Transications on Signal Processing, 2003,51(10):2592-2601

[14] Li X R. A Survey of maneuvering target tracking. part I dynamic models. IEEE Transactions on Aerospace and Electronic Systems, 2003, 39(4): 1333-1364. 